\title{
Selenoprotein $P$ and glutathione peroxidase $(E C$ 1.11.1.9) in plasma as indices of selenium status in relation to the intake of fish
}

\author{
BY W. HUANG ${ }^{1}$, B. ÅESSON ${ }^{1 *}$, B. G. SVENSSON ${ }^{2}$, A. SCHÜTZ $Z^{2}$, R. F. BURK ${ }^{3}$ \\ AND S. SKERFVING ${ }^{2}$ \\ ${ }^{1}$ Department of Applied Nutrition and Food Chemistry, and ${ }^{2}$ Department of Occupational and \\ Environmental Medicine, University of Lund, Lund, Sweden \\ ${ }^{3}$ Division of Gastroenterology, Department of Medicine, Vanderbilt University, Nashville, TN, USA
}

(Received 25 February 1994 - Revised 29 June 1994-Accepted 7 July 1994)

\begin{abstract}
In Sweden fish is considered to be an important source of dietary Se. Therefore Se status was assessed in forty-one middle-aged men with widely varying fish consumption. Glutathione peroxidase (EC 1.11.1.9) and selenoprotein $P$ in plasma were measured by radioimmunoassay. Plasma Se among the men increased slightly with increasing consumption of fish, but no such increases in the concentrations of glutathione peroxidase and selenoprotein $P$ in plasma were observed. Moreover, no correlation was found between plasma Se and glutathione peroxidase or selenoprotein $P$. Instead, glutathione peroxidase was significantly correlated with selenoprotein $P(r 0.73, P<0.001)$, indicating that both glutathione peroxidase and selenoprotein $P$ were functional indicators of Se status in this group. The proportion of plasma Se located in glutathione peroxidase decreased with increasing plasma Se. The results suggest that the Se consumed from fish had no apparent effect on the amount of Se incorporated into the functional selenoproteins of plasma. It is concluded that in some cases selenoproteins may be better biological markers of Se status than the total concentration of Se.
\end{abstract}

Dietary fish intake: Glutathione peroxidase: Selenoprotein P: Selenium

Fish is an important component of the diet, being rich in protein, $n-3$ polyunsaturated fatty acids and certain trace elements and vitamins. In Finland it was calculated that in 1980 about one third of the dietary Se was provided from fish (Koivistoinen, 1980). Less is known about the bioavailability and the metabolic fate of Se from dietary fish. Previous studies showed that subjects with high consumption of fish had higher concentrations of Se in plasma than those with low intake (Svensson et al. 1992). Moreover, a change to a diet rich in fish increased the Se concentration in plasma (Robinson et al. 1978; Thorngren \& Åkesson, 1987). Several studies have shown an association between Se concentration and glutathione peroxidase (EC 1.11.1.9) activity in plasma of healthy adults (Åkesson \& Steen, 1987; McMaster et al. 1990), but little is known of the relationship of fish intake to specific selenoproteins. In addition to extracellular glutathione peroxidase, selenoprotein $P$ has been demonstrated in plasma (Takahashi et al. 1987; Burk, 1991; Åkesson et al. 1994). In the present study glutathione peroxidase and selenoprotein $\mathbf{P}$ in plasma were studied in relation to consumption of fish, using newly developed radioimmunoassay methods. 


\section{METHODS}

Study participants

From an earlier study group (Svensson et al. 1992) drawn from three rural areas of southeast Sweden, forty-one middle aged men were selected because of their differing levels of fish consumption. Twenty were classified as high, twelve as moderate and nine as nonconsumers of fish, having mean weekly intakes of 1160,375 and $0 \mathrm{~g}$. Intakes were calculated from completed questionnaires as already described (Svensson et al. 1992).

For comparison, Se status was also studied among sixteen patients with a very wide variation in serum Se concentration $(0 \cdot 1-2 \cdot 9 \mu \mathrm{mol} / \mathrm{l})$ as described previously (Huang \& Åkesson, 1993). This heterogeneous group was not characterized further and was selected just to indicate changes in selenoprotein levels at more than ten-fold variation in serum Se.

\section{Analytical methods}

The radioimmunoassay of glutathione peroxidase and selenoprotein $\mathbf{P}$ has been described elsewhere (Huang \& Akesson, 1993; Åkesson et al. 1994). The measurement of Se has also been described previously (Svensson et al. 1992).

\section{Calculations}

Correlations were calculated as linear correlation coefficients and Spearman's $\rho$. One-way ANOVA and Student's $t$ test were used for evaluation of differences among groups.

\section{RESULTS}

\section{Selenoproteins and selenium in plasma}

The concentration of Se in plasma varied among the groups consuming different amounts of fish (Table 1). It was higher in the group with the high intake of fish compared with the groups with no or moderate consumption. On the other hand, the concentrations of selenoprotein $\mathbf{P}$ and glutathione peroxidase did not differ significantly among the three groups.

As reported previously (Svensson et al. 1992), plasma Se was correlated with the intake of fish and also with the proportion of $n-3$ polyunsaturated fatty acids in plasma phosphatidylcholine. In the present study no correlation between either selenoprotein $P$ or glutathione peroxidase and the intake of total fish, lean fish or oily fish was found. Neither of the two selenoproteins was correlated with the proportion of $n-3$ fatty acids, which varied several-fold among the groups (Svensson et al. 1993).

\section{Relationship between selenoproteins and selenium in plasma}

The correlation between selenoprotein $\mathrm{P}$ and Se was -0.29 (Fig. 1). Similarly, plasma glutathione peroxidase had no positive correlation with plasma Se $(r-0-15)$ (Fig. 2), suggesting that the Se incorporated into plasma selenocompounds due to a large consumption of fish was not primarily incorporated into the selenoproteins. Interestingly enough, plasma glutathione peroxidase was correlated with selenoprotein $\mathrm{P}(r 0.73$, $P<0.001$; Fig. 3), indicating that although the two indices were not positively related to plasma Se, both reflected another aspect of Se status, maybe functionally active selenoproteins. The association was apparently similar in all three groups, and moreover the data did not suggest that the Se requirement of any of the selenoproteins had reached a saturation level (Fig. 3).

The proportion of plasma Se located in glutathione peroxidase was calculated according to its structure as described previously (Huang \& A kesson, 1993). This proportion 
Table 1. Selenium and selenoprotein levels in the plasma of subjects consuming different amounts of fish $\dagger$

(Mean values with their standard deviations)

\begin{tabular}{|c|c|c|c|c|c|c|}
\hline & \multicolumn{6}{|c|}{ Fish consumption } \\
\hline & \multicolumn{2}{|c|}{ None $(n 9)$} & \multicolumn{2}{|c|}{ Moderate ( $n$ 12) } & \multicolumn{2}{|c|}{ High $(n 20)$} \\
\hline & Mean & SD & Mean & SD & Mean & SD \\
\hline $\mathrm{Se}(\mu \mathrm{mol} / \mathrm{l})$ & 0.99 & 0.12 & 1.01 & 0.11 & $1 \cdot 17^{* *}$ & 0.15 \\
\hline GSHPx (mg/l) & 3.51 & 1.07 & $3 \cdot 61$ & 0.85 & $3 \cdot 67$ & 1.04 \\
\hline Se \%-GSHPx $\ddagger$ & $16 \cdot 7$ & $5 \cdot 1$ & $16 \cdot 7$ & $4 \cdot 2$ & $15 \cdot 0$ & $5 \cdot 3$ \\
\hline SeP (arbitrary units) & 1.61 & 0.44 & 1.51 & 0.26 & 1.49 & 0.38 \\
\hline
\end{tabular}

GSHPx, glutathione peroxidase (EC 1.11.1.9); SeP, selenoprotein $P$.

** Mean value was significantly different from those for moderate and zero consumers, $P<0.01$.

$\dagger$ For details of subjects and procedures, see p. 456.

$\$$ Percentage of plasma Se located in glutathione peroxidase.

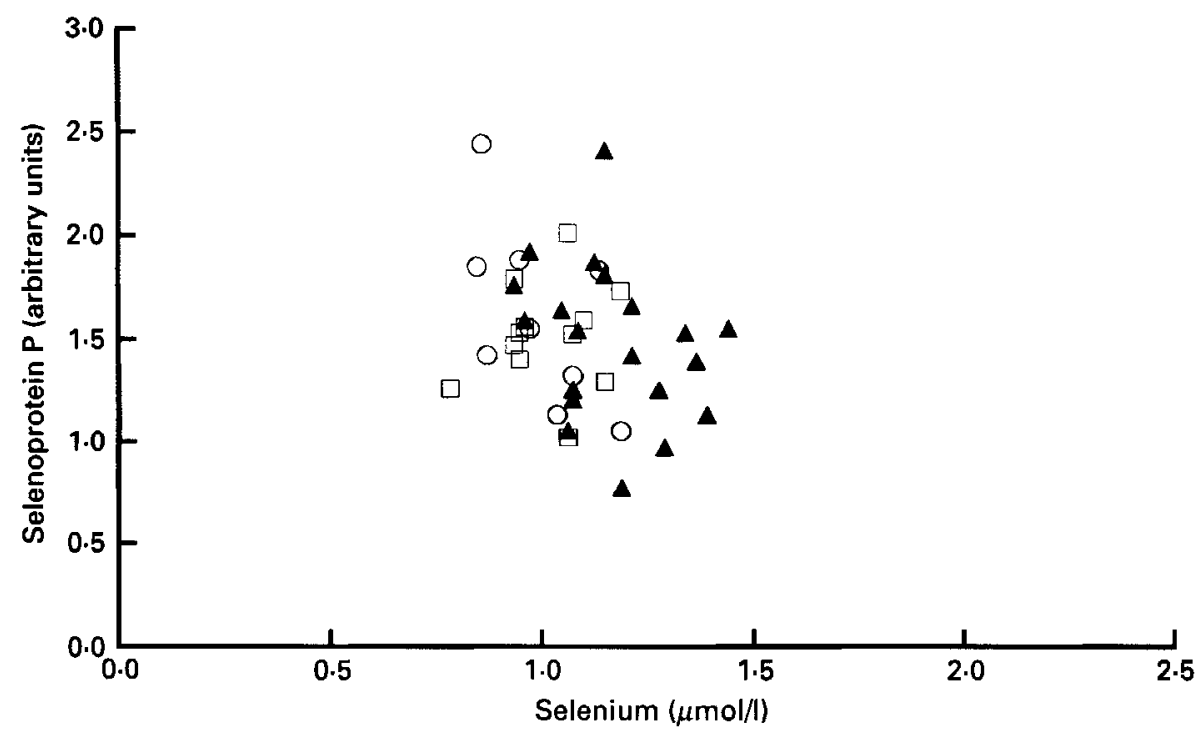

Fig. 1. Relationship between plasma selenium and selenoprotein $\mathbf{P}$ concentrations among men with high $(\mathbf{\Delta})$, moderate $(\square)$ or no $(O)$ fish consumption $(r-0.29, P=0.07 ; \rho-0.28, P=0.08)$.

decreased with increasing plasma Se concentration $(r-0 \cdot 56, P<0.001)$. The (selenoprotein $P)$ : Se ratio was calculated as a measure of the proportion of Se located in selenoprotein $P$, and this variable was also negatively correlated with total Se concentration in plasma $(r-0.66, P<0.001)$. These negative associations were similar also within the three groups. The mean percentage of plasma Se located in glutathione peroxidase tended to be lower in the high consumer group. A similar negative association between (selenoprotein P): Se and plasma Se was observed in a study of healthy adults (Marchaluk et al. 1995).

Previous studies of the cohort showed positive correlations between Se in plasma and $\mathrm{Hg}$ in blood, plasma and erythrocytes (Svensson et al. 1992). In the present study, neither 


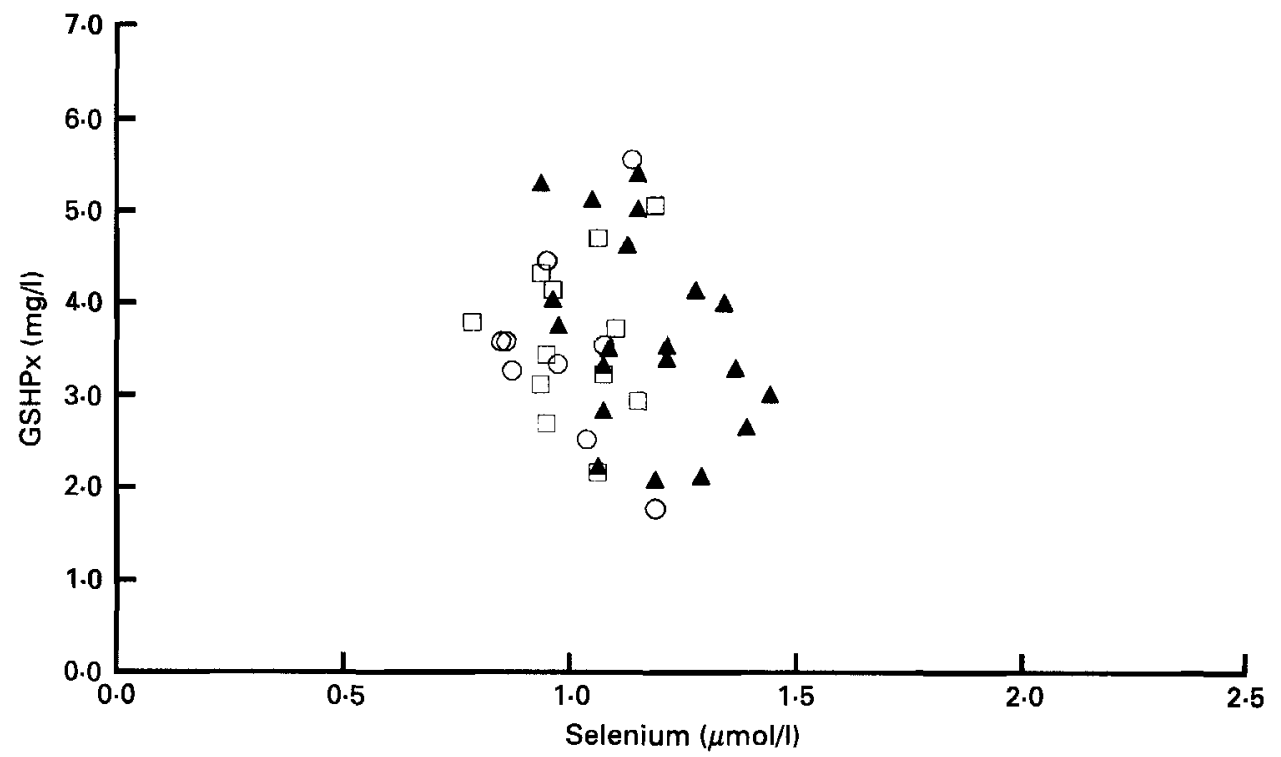

Fig. 2. Relationship between plasma selenium and glutathione peroxidase protein (GSHPx; EC 1.11.1.9) concentrations among men with high $(\Delta)$, moderate $(\square)$ or no $(O)$ fish consumption $(r-0.15, P=0.36$; $\rho-0.17, P=0.28$ ).

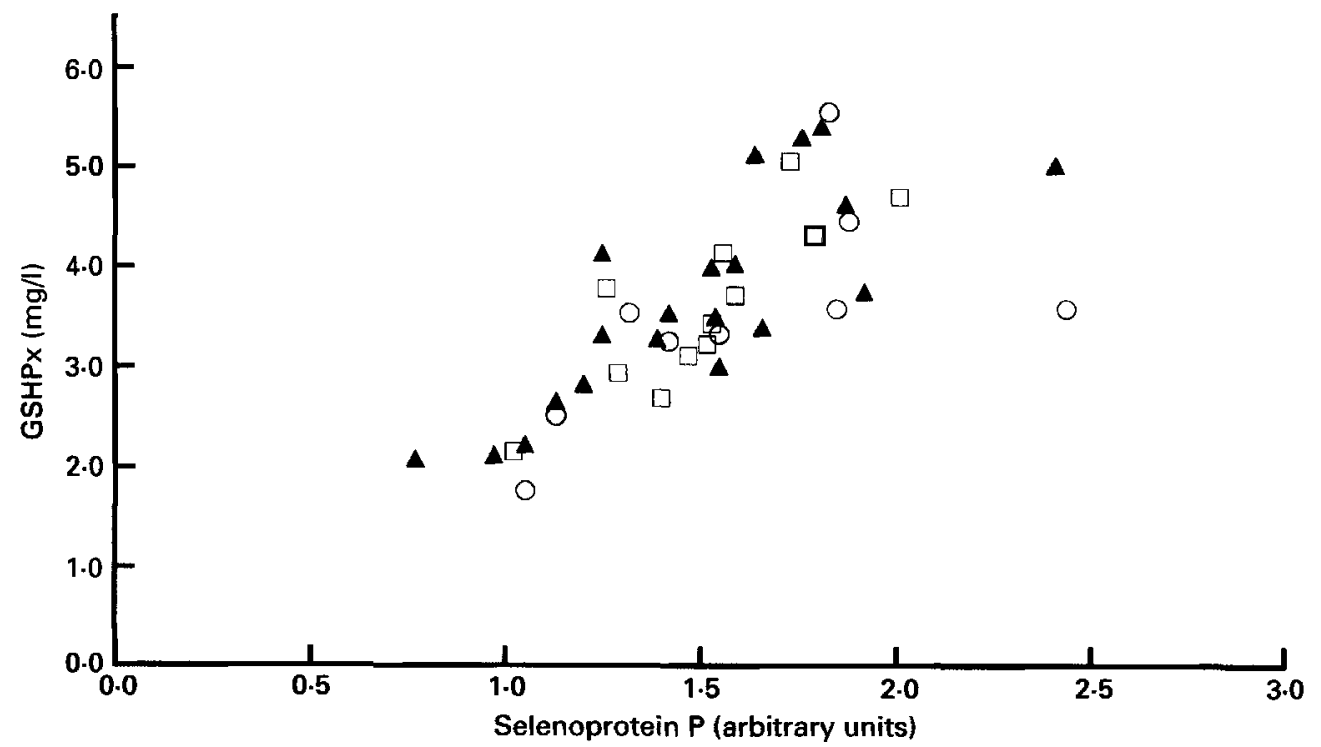

Fig. 3. Relationship between plasma selenoprotein $P$ and glutathione peroxidase protein (GSHPx; EC 1.11 1.9) concentrations among men with high $(\Lambda)$, moderate $(\square)$ or no $(O)$ fish consumption $(r 0 \cdot 73, P<0 \cdot 001 ; \rho 0 \cdot 79$, $P<0.001)$.

plasma glutathione peroxidase nor selenoprotein $\mathbf{P}$ was significantly associated with the concentration of $\mathrm{Hg}$ in plasma, blood, erythrocytes or urine.

For comparison, Se status in a heterogeneous group of patients with widely varying serum Se concentration was studied (Fig. 4). Among these samples selenoprotein $P$ was 

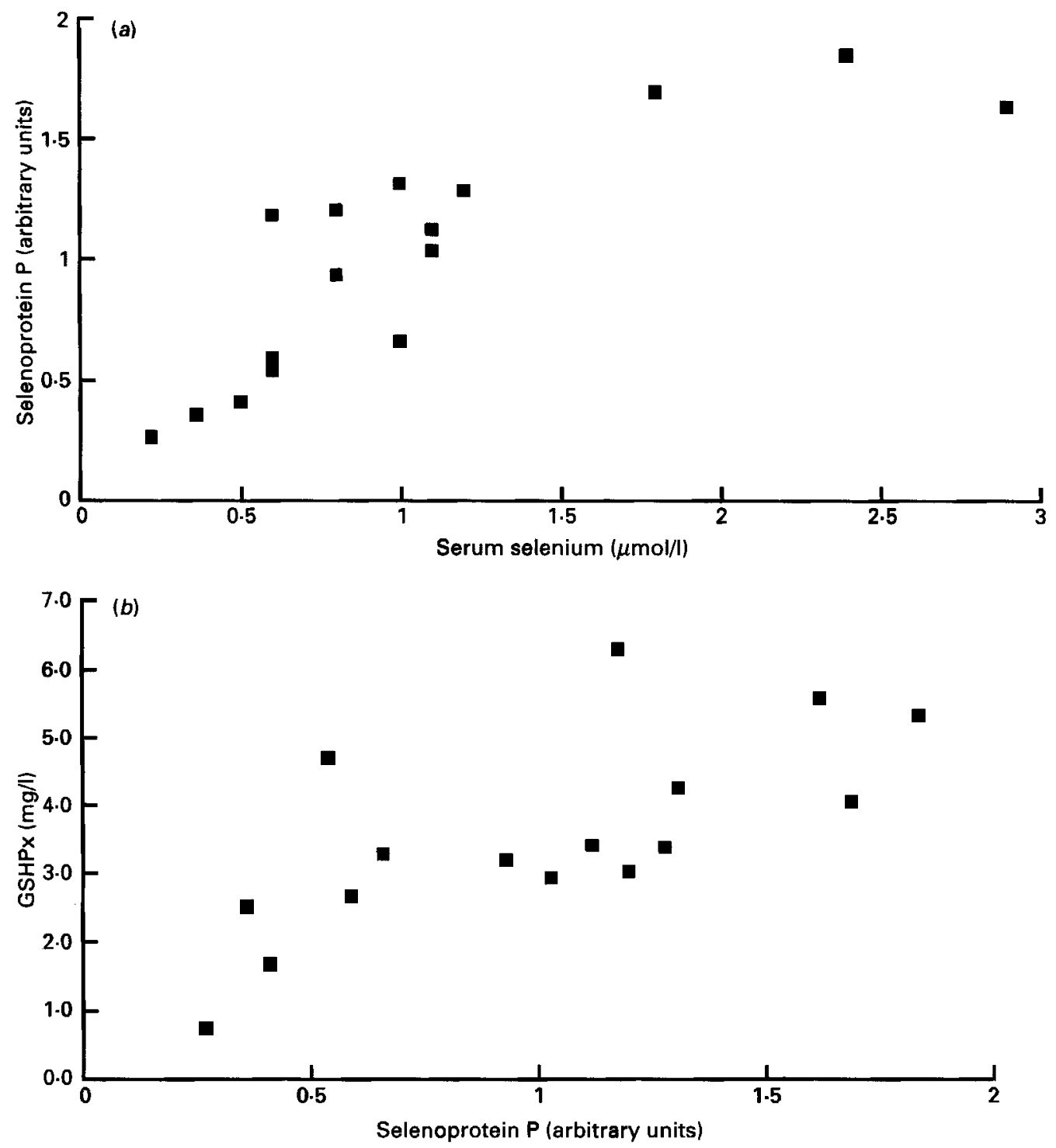

Fig. 4. Associations of plasma selenoprotein $\mathrm{P}$ with $(a)$ selenium $(r 0.83, P<0.001)$ and $(b)$ glutathione peroxidase protein (GSHPx; EC 1.11.1.9) $(r 0.69, P=0.003)$ in patients with widely varying serum selenium concentrations.

significantly correlated both with total Se $(r 0.83)$ and with glutathione peroxidase protein ( $r$ 0.69). Similarly, in a group of healthy adults with a more narrow range of serum Se levels the correlation between Se and selenoprotein $P$ was 0.68 (Marchaluk et al. 1995).

\section{DISCUSSION}

Previous studies have shown that plasma Se and glutathione peroxidase activity are positively correlated in healthy subjects with Se status typical for Europe (Akesson \& Steen, 1987; McMaster et al. 1990). A similar association was found using an immunochemical 
assay of plasma glutathione peroxidase (Huang \& Åkesson, 1993). Data in Fig. 4 show a positive correlation also between Se and selenoprotein $\mathbf{P}$ in plasma, and similar findings emerge from an ongoing study of healthy European subjects (Marchaluk et al. 1995).

The lack of a positive correlation between plasma Se and the two selenoproteins in the men consuming different amounts of fish might have several explanations. It is possible that some of the Se absorbed from ingested fish was in a molecular form that was not utilized for selenoprotein synthesis, but instead accumulated in other compounds, e.g. proteins containing unspecifically incorporated Se (McConnell \& Hoffman, 1972). One such possible dietary form of Se is selenomethionine (Yasumoto et al. 1988), but this compound has so far not been demonstrated in fish.

Animal studies have indicated that the bioavailability of Se in certain kinds of fish was less than that in other foods (Mutanen, 1986). The bioavailability of Se from different fish in humans has not been studied in detail. One study suggested that the increase in plasma Se after switching to a diet rich in fish was smaller than would have been expected (Thorngren \& Åkesson, 1987). Moreover, Meltzer et al. (1993) found that a diet rich in fish, containing $50 \%$ more Se than a control diet, did not significantly affect serum and platelet Se levels in healthy subjects.

The forms of Se in fish have not been thoroughly studied. Chromatographic studies suggest that the Se compounds in fish differ from those in meat (̊̊kesson \& Srikumar, 1994). Such differences may be one factor explaining differences in Se bioavailability from different foods.

Another possible explanation for the absence of association between fish intake and selenoprotein levels may be that the higher consumption of methylmercury by high fish consumers, as reflected in their higher concentration of $\mathrm{Hg}$ in blood, plasma and erythrocytes (Svensson et al. 1992), may have interfered with the utilization of Se for selenoprotein biosynthesis. Several reports, especially from studies in animals, indicate that $\mathrm{Se}$ interacts with both inorganic $\mathrm{Hg}$ and methylmercury, although detailed mechanisms for such interactions are not known (Burk et al. 1977; Magos, 1991). In the present study no correlation was found between plasma selenoproteins and $\mathrm{Hg}$ in plasma, blood, erythrocytes, and urine, thus giving no support for an interaction between $\mathrm{Hg}$ from fish intake and selenoproteins.

Se status affected the distribution of plasma Se among glutathione peroxidase and other proteins. The calculated proportion of Se associated with glutathione peroxidase was inversely correlated with plasma Se among the men with varying fish intake. Gel filtration experiments indicated that the distribution of Se among plasma proteins was affected after supplementation with protein-bound Se (Borglund \& Akesson, 1988). In conclusion, the present study suggests that at least in some cases plasma selenoproteins may be better markers of Se status than the total concentration of Se.

The study was supported by grants from the National Swedish Environmental Protection Agency, the Swedish Medical Research Council (project no. 9873), the Swedish Council for Forestry and Agricultural Research, the Påhlsson Foundation, the Swedish Nutrition Foundation, and the Medical Faculty of the University of Lund. Skilful technical assistance was given by Ms Anita Nilsson and Ms Marianne Nilsson.

\section{REFERENCES}

Åkesson, B., Bellew, T. \& Burk, R. F. (1994). Purification of selenoprotein P from human plasma. Biochimica et Biophysica Acta 1204, $243-249$.

Åkesson, B. \& Srikumar, T. S. (1994). Occurrence of low-molecular-weight and high-molecular-weight selenium compounds in fish. Food Chemistry 51, 45-49. 
Åkesson, B. \& Steen, B. (1987). Plasma selenium and glutathione peroxidase in relation to cancer, angina pectoris and short-term mortality in 68-year-old men. Comprehensive Gerontology 1A, 61-64.

Borglund, M. \& Åkesson, B. (1988). Effect of selenium supplementation on the distribution of selenium in plasma proteins of healthy subjects. International Journal for Vitamin and Nutrition Research 58, 97-102.

Burk, R. F., Jordan, H. E. Jr \& Kiker, K. W. (1977). Some effects of selenium status on inorganic mercury metabolism in the rat. Toxicology and Applied Pharmacology 40, 71-82.

Burk, R. F. (1991). Molecular biology of selenium with implications for its metabolism. FASEB Journal 5, 2274-2279.

Huang, W. \& Åkesson, B. (1993). Radioimmunoassay of glutathione peroxidase in human serum. Clinica Chimica Acta 219, 139-148.

Koivistoinen, P. (ed.) (1980). Mineral element composition of Finnish foods: N, K, Ca, Mg, P, S, Fe, Cu, Mn, $\mathrm{Zn}, \mathrm{Mo}, \mathrm{Co}, \mathrm{Ni}, \mathrm{Cr}, \mathrm{F}, \mathrm{Se}, \mathrm{Si}, \mathrm{Rb}, \mathrm{Al}, \mathrm{B}, \mathrm{Br}, \mathrm{Hg}, \mathrm{As}, \mathrm{Cd}, \mathrm{Pb}$ and ash. Acta Agricultura Scandinavica 22, Suppl., $1-171$.

McConnell, K. P. \& Hoffman, J. L. (1972). Methionine-selenomethionine parallels in rat liver polypeptide chain synthesis. FEBS Letters 24, 60-62.

McMaster, D., Bell, N., Anderson, P. \& Love, A. H. G. (1990). Automated measurement of two indicators of human selenium status, and applicability to population studies. Clinical Chemistry 36, 211-216.

Magos, L. (1991). Overview of the protection given by selenium against mercurials. In Advances in Mercury Toxicology, pp. 289-298 [T. Suzuki, N. Imura and T. W. Clarkson, editors]. New York: Plenum Press.

Marchaluk, E., Persson-Moschos, M., Thorling, E. B. \& Åkesson, B. (1995). Variation in selenoprotein P concentration in serum from different European regions. European Journal of Clinical Nutrition (In the Press).

Meltzer, H. M., Bibow, K., Paulsen, I. T., Mundal, H. H., Norheim, G. \& Holm, H. (1993). Different bioavailability in humans of wheat and fish selenium as measured by blood platelet response to increased dietary Se. Biological Trace Element Research 36, 229-241.

Mutanen, M. (1986). Bioavailability of selenium. Annals of Clinical Research 18, 48-54.

Robinson, M. F., Rea, H. M., Friend, G. M., Stewart, R. D. H., Snow, P. C. \& Thomson, C. D. (1978). On supplementing the selenium intake of New Zealanders. 2. Prolonged metabolic experiments with daily supplements of selenomethionine, selenite and fish. British Journal of Nutrition 39, 589-600.

Svensson, B. G., Åkesson, B., Nilsson, A. \& Skerfving, S. (1993). Fatty acid composition of serum phosphatidylcholine in healthy subjects consuming varying amounts of fish. European Journal of Clinical Nutrition 47, 132-140.

Svensson, B. G., Schütz, A., Nilsson, A., Åkesson, I., Åkesson, B. \& Skerfving, S. (1992). Fish as a source of exposure to mercury and selenium. The Science of the Total Environment 126, 61-74.

Takahashi, K., Avissar, N., Whitin, J. \& Cohen, H. (1987). Purification and characterization of human plasma glutathione peroxidase: a selenoglycoprotein distinct from the known cellular enzyme. Archives of Biochemistry and Biophysics 256, 677-686.

Thorngren, M. \& Åkesson, B. (1987). Effect of dietary fish on plasma selenium and its relation to haemostatic changes in healthy adults. International Journal for Vitamin and Nutrition Research 57, 429-435.

Yasumoto, K., Suzuki, T. \& Yoshida, M. (1988). Identification of selenomethionine in soybean protein. Journal of Agricultural and Food Chemistry 36, 463-467. 